\title{
RISK ASSESSMENT OF MANUFACTURING AND USAGE OF TEST SYSTEMS FOR QUALITY CONTROL OF COMPOUNDED PREPARATIONS
}

\author{
Vadim Prokopets, Oleksandr Zdoryk, Viktoria Georgiyants \\ Department of Pharmaceutical Chemistry, National University of Pharmacy, \\ Kharkov, Ukraine
}

\begin{abstract}
INTRODUCTION: The development and implementation of test systems in pharmacy practice could be one of the ways of optimization of the quality control of compounded preparations. Test kits, which have been manufactured in a pharmacy, are simple, cheap and effective for the quality control of a wide range of pharmaceutical preparations. Guidelines for quality must be taken into account during the production of such test kits to enable a pharmacist-analyst to fully perform his tasks using these analytical tools.

The aim of the work is to analyze the risks that may occur during the lifecycle of test systems; to establish the risks that have the biggest impact on quality control of compounded preparations with these analytical tools and to determine the major ways to minimize the impact of risks at all stages of work with test systems.

MATERIALS AND METHODS: Ishikawa diagrams and Failure Mode, Effects and Criticality Analysis (FMECA) were used to analyze the risks during the lifecycle of test systems. Data were received through "brainstorming session" and questioning respondents that were related to the development and practical use of these analytical tools.

RESULTS: According to the results Risk Priority Number (RPN) for each of the stages of the lifecycle of the test systems has been calculated. The highest values of RPN are observed at the stages of manufacturing of the test systems (54.95\%), the quality control (21.93\%) and the use of the test systems (18.66 \%). Each of the stages of the lifecycle of the test system consists of a number of phases; the most important among them are: preparation of the reagent solution $-20.24 \%$; chemical analysis $-17.93 \%$; visual fixation of results $-9.21 \%$ and training of the personnel $-8.89 \%$.

CONCLUSIONS: The recommendations concerning minimization of risk throughout the lifecycle of test kits were developed relying on the results. Optimization in accordance with these guideline procedures of manufacturing, quality control, storage and using these test systems allows to ultimately get high-quality analytical tools for the quality control of compounded preparations.
\end{abstract}

Keywords: chemical test kits, compounded preparations, quality control, risk, valuation

Address for correspondence:

Vadim Prokopets

National University of Pharmacy

53 Pushkinska St.,

Kharkov, Ukraine, 61000

e-mail:wolf_prokopetz@ukr.net

Received: November 23, 2016

Accepted: December 21, 2016

\section{INTRODUCTION}

Quality control of the compounded preparations is not the only uncontested mechanism of ensuring the quality of medicines. Methods of risk analysis and management in the pharmaceutical industry are implemented in many countries $(1,2)$, including Ukraine, in accordance with the guideline ICH-Q9 «Quality Risk Management". The recom- 
mendations of this guideline can be applied not only to the pharmaceutical companies, but also in the pharmacies, which are engaged in compounding and dispensing preparations (3).

With the purpose of express analysis of compounded preparations test systems containing heavy metals were developed. In previous publications the use of test kits based on filter paper and modified with reagents has been discussed (4-6). Using such test systems and physical immobilization of reagents makes the preparation of test kits easily available in pharmacies. Creation of simple, cheap and standardized test systems, with reagents that worked well during the macroanalysis is important and can greatly simplify the process of in-pharmacy quality control. Implementation of new processes - preparation of test systems in compounding pharmacies, making mistakes can lead to the incorrect evaluation of the quality of compounded preparations. Test systems are the tools used for quality control of medicines so the requirements for analysis and risk management can be extended to all stages of the lifecycle of these analytical tools.

The aim of the work is to analyze the risks that may occur during the lifecycle of test systems; to establish the risks that have the biggest impact on quality control of compounded preparations with these analytical tools and to determine the major ways to minimize the impact of risks at all stages of working with test systems.

\section{MATERIALS AND METHODS}

Potential risks and ways to avoid them during the lifecycle of test system based on filter paper impregnated with the salts of heavy metals $\mathrm{FeCl}_{3}$, $\mathrm{CuSO}_{4}, \mathrm{CoCl}_{2}$ are discussed in the article. Methods concerning manufacturing, storage and using these test systems were described in previous publications (4-6).

The following tools were used to analyze the risks during the lifecycle of test systems: Ishikawa diagrams or Fishbone diagrams and Failure Mode, Effects and Criticality Analysis (FMECA).

Ishikawa's diagrams have wide application in various fields of human activity; they can also be effectively used in pharmacy, including in the work of a pharmacy shop $(7,8)$. The analysis of potential risks was conducted using the method of "brain- storming", the main stages of the life cycle of test systems have been allocated with further distribution of these risks. The Ishikawa's diagrams were constructed with attraction of specialists who participated in the designing, manufacturing and use of these analytical tools.

Failure Mode, Effects and Criticality Analysis (FMECA) has found application in pharmacies in Europe (9). This method of analysis was important for us because of the possibility of digital expression of risks - determining Risk Priority Number - (RPN). RPN is the product probability of occurrence of risk $(\mathrm{O})$, severity of risk $(\mathrm{S})$ and probability of detection of risk (D), calculated for each individual risk. To assess each factor a 10 -point scale provided in (8) has been used. The priority number of risk was calculated using the following formula based on the data $(7,10)$ :

$$
\mathrm{RPN}=\mathrm{S}^{\star} \mathrm{O}^{\star} \mathrm{D}
$$

The data were obtained by questioning respondents - pharmacists and laboratory technician assistants who participated in the development, manufacturing, quality control and use of test-systems described in (4-6). 30 specialists were interviewed.

\section{RESULTS AND DISCUSSION}

\section{Ishikawa's diagrams or Fishbone diagrams}

The factors that have a negative impact on the quality control of compounded preparations by using the test kits reflect in the Ishikawa diagram on Figure 1. The risks are distributed in 4 stages: the risks of manufacturing, quality control, use and storage of the test kits; the impact of personnel training is presented separately, as it has influence on all stages of the lifecycle of test kits.

The largest group of risks is the risks that impact the process of manufacturing of the test kits. We have represented it separately in the Figure 2.

Figure 1 and Figure 2 show the stage of the manufacturing of test systems has the largest number of risks of the first and second order. This situation is associated with a large number of operations at this stage (Table 1). 


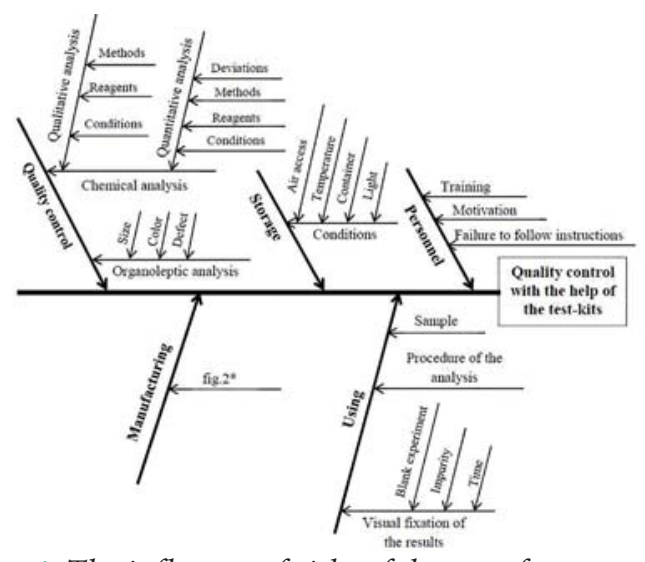

Figure 1. The influence of risks of the use of test systems on the quality control of compound preparations

Failure Mode, Effects and Criticality Analysis

The risks and their possible effects were analyzed in more details by questioning the specialists, followed by the processing of data using FME$\mathrm{CA}$. The results of the questioning about the risks and their consequences that may occur at each phase of all 4 stages of the lifecycle of test systems are presented in Table. 1. The 35 risks were selected, some of which are repeated, as they are typical for different phases and have different effects on each of them. In particular, this applies to training of the personnel that, unlike Ishikawa diagrams, is not submitted in a separate group. The absolute and relative values of RPN for each risk at all stages of the lifecycle of the test system are presented in Table 1.

The percentage of impact of each stage and phase on the overall value of RPN was calculated after statistical processing of the questionnaires. The results are presented in Table 2.

Table 1 shows: the manufacturing of the test systems has the highest rate of RPN $-54.95 \%$, due to the large number of phases and the risks at each phase; the quality control and the use of the test systems also make a significant contribution in the overall value of RPN: RPN (Quality control) - 21.93\%, RPN (Using) - 18.66\%. Selected stages contain different number of phases, and as result, different number of risks, and so more detailed look should be taken at the risks that may occur at each phase of the lifecycle of the test systems:

$\diamond$ preparation of the reagent solution - $20.24 \%$; this high index is caused by a large number of

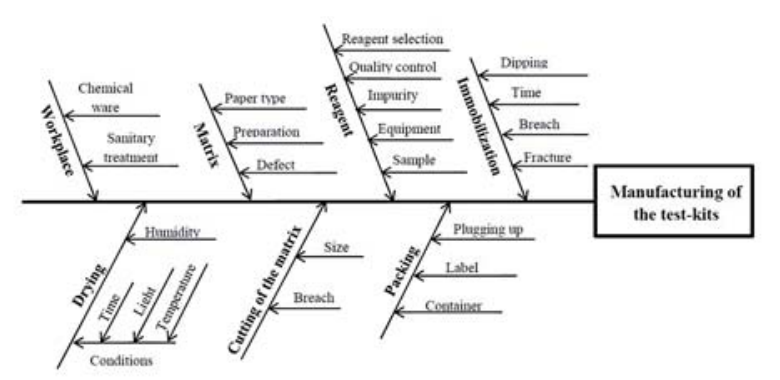

Figure 2. The risks that impact the process of manufacturing of test systems

operations, errors of which have a high threat to receive a defective test system;

$\diamond$ chemical analysis - 17.93\%; the risks that occur at this phase include the use of invalidated analysis methods and reagents that do not comply with the requirements of pharmacopoeias, incorrect concentration of the reagent on the surface of the test system, which can cause narrowing of the range of application of a test system;

$\diamond$ visual fixation of results has RPN of 9.21\%. Failure to comply with the time period of observation, the absence of blank experiment and the problems with fixing results (presence of impurities, which can be able to disguise the analytical effect of the studied component) are the main risks at the this phase;

$\diamond$ training of the personnel, this factor has a low value of RPN at each of the stages, but the total value of "human factors" is $8.89 \%$, which indicates the inadmissibility of ignoring the increase of the quality of the personnel training;

$\diamond$ the physical immobilization is an important phase (8.54\%) - errors, which take place at this phase can influence the concentration of reagents at the surface of test-system and expiration date;

$\diamond$ the phases of preparation of filter paper (7.94\%) and analysis (4.73\%) have high index of RPN, because of the defects of filter papers and the realization of experiments without blank experiment, respectively;

$\diamond$ packaging and labeling of the test systems (5.79\%) and control of the storage conditions (3.18\%) also require increased attention, risks at these phas- 
Vadim Prokopets, Oleksandr Zdoryk, Viktoria Georgiyants

Table 1. Assessment of occurrence, severity and detection of the risks throughout the lifecycle of test kits

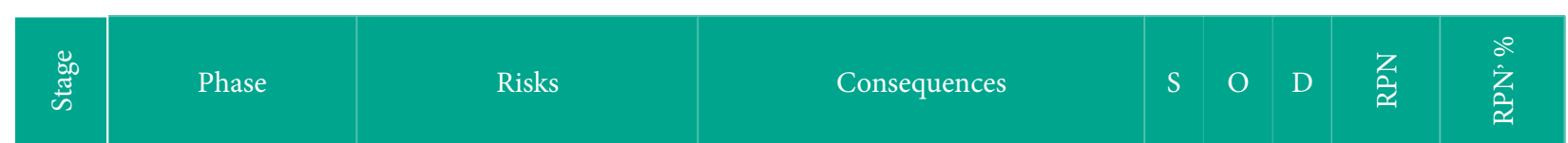

1. Unsatisfactory sanitary Chemical contamination of test treatment of rooms, system. Invalid results of the $\begin{array}{llll}5 & 3 & 4 & 60\end{array}$ equipment, personnel analysis.

1.1. Preparation of the workplace

2. Wrongly selected chemical ware

1.2. Preparation of filter paper sheets

1.2.1. Selection of paper type

1. Paper defect

1.2.2. Cutting paper into sheets of a specified size $\left(50^{\star} 80 \mathrm{~mm}\right)$

1. Inappropriate number of strips from one sheet 1. Error during reagent selection

2. Error during sampling
Inappropriate concentration of reagent. Increased time for manufacturing of solution of the desired concentration (adjusting the concentration and repeated quantitative determination).

Invalid results of the analysis (high probability of false positive or false negative result). Incorrect concentration of the reagent on the surface of the test-system. Reduction of the test system 's expiration date. The test system is unusable.

Increasing expenses and time for production of one test system. Different concentrations of the reagent on different strips from the same sheet. Invalid results of the analysis.

1.3. Preparation of reagent solution (as required by State Pharmacopoeia of Ukraine)

1.3.1. Sampling

3. Using not appropriately calibrated equipment

4. Ignoring the moisture content, impurities

1.3.2. Dissolution and removing 1. Wrongly selected chemical ware

the solvent to the mark

2. Errors during removing the solvent to the mark

1. Using a invalidated analysis methods and reagents that do not

1.3.3. Quality control of the comrespond the requirements pound solution of pharmacopoeias

2. Inconsistency in terms of sampling, analysis, recording results
The test system is unusable.

Inappropriate concentration

of reagent. Increased time for manufacturing of solution with the desired concentration (adjusting the concentration and repeated quantitative determination).

Decrease of the quality of analysis. Invalid results of the analysis. Increasing expenses and time for production of test systems $\begin{array}{llll}5 & 3 & 5 & 75\end{array}$

$\begin{array}{llll}6 & 5 & 5 & 150\end{array}$

$\begin{array}{llll}8 & 4 & 3 & 96\end{array}$

$\begin{array}{llll}6 & 3 & 4 & 72\end{array}$

$\begin{array}{llll}6 & 3 & 4 & 72\end{array}$

$\begin{array}{llll}6 & 4 & 4 & 96\end{array}$

$\begin{array}{llll}5 & 3 & 4 & 60\end{array}$

$\begin{array}{llll}5 & 3 & 4 & 60\end{array}$

$\begin{array}{llll}8 & 4 & 4 & 128\end{array}$

$\begin{array}{llll}7 & 3 & 4 & 84\end{array}$ 

the coverage level and area of the sheet

\subsubsection{Control of} the time of immobilization
1.4.1. Control of

1. Partial immersion of a sheet in a solution

2. The inflection of sheets

3. The breach of sheet parts

1. Incorrect concentration of the reagent on the surface of the test-system

1.5. Drying the test system

\subsubsection{Control of}

the remainder of the moisture

1.5.2. Control of the drying conditions

1.6. Cutting of sheet into the teststrips

1.7. Packaging and labeling of testsystems

\section{Excess of moisture}

1. Failure to comply drying conditions (time, light, temperature)

1. Contradiction of size to that specified in the documentation $(50 * 5 \mathrm{~mm})$

2. The break of sheet

1. Inappropriate storage containers

2. Bad sealing containers

3. Preparation of labels

1.8. Training of

1. Unsatisfactory quality of training

1. Defect (size, color intensity, damage of a testsystem)

analysis lysis

2.2. Chemical analysis

1. Using invalidated analysis methods and reagents that do not respond the

2.2.1. Qualitative analysis copoeias

2. Inconsistency in terms of sampling, analysis, fixing results

Different concentrations of the

reagent on different strips from the same sheet. Invalid results of the analysis.

Inappropriate number of strips from one sheet.

Invalid results of the analysis. Reduction of the test system 's expiration date.

Errors of the separation of sheets into strips. The break of testsystems.

Reduction of the test system `s expiration date. Invalid results of the analysis.

Inappropriate number of strips from one sheet. Invalid results of the analysis. The test system is unusable.

Reduction of the test system 's expiration date. Invalid results of the analysis. The test system is unusable.

False labeling.

Invalid results of the analysis. The test system is unusable. Reduction of the test system 's expiration date. Decrease of the quality of analysis. Invalid results of the analysis. Increasing expenses and time for production of test-systems.

Invalid results of the analysis. The test system is unusable.

Decrease of the quality of analysis. Invalid results of the analysis. Increasing expenses and time for production of test-systems. The test system is unusable. $\begin{array}{llll}6 & 4 & 2 & 48\end{array}$

$\begin{array}{lllll}8 & 4 & 3 & 96 & 2.91 \\ 5 & 3 & 4 & 60 & 1.82 \\ 5 & 2 & 3 & 30 & 0.91 \\ 6 & 4 & 4 & 96 & 2.91\end{array}$

$\begin{array}{lllll}4 & 3 & 4 & 48 & 1.45\end{array}$

$\begin{array}{lllll}4 & 2 & 3 & 24 & 0.73\end{array}$

$\begin{array}{lllll}5 & 2 & 3 & 30 & 0.91\end{array}$

$\begin{array}{lllll}8 & 3 & 3 & 72 & 2.18\end{array}$

$\begin{array}{lllll}7 & 4 & 2 & 56 & 1.70\end{array}$

$\begin{array}{lllll}7 & 3 & 3 & 63 & 1.91\end{array}$

$\begin{array}{lllll}7 & 4 & 3 & 84 & 2.54\end{array}$

$\begin{array}{lllll}7 & 4 & 4 & 112 & 3.39\end{array}$ 
2.2.2. Quantitative analysis

2.3 Training of the personnel

范

3.1. Control of the storage conditions

\subsection{Training of the personnel}

4.1. Sampling of substances

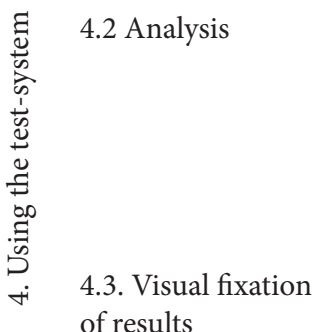
of results

4.4. Training of the personnel
1. Using invalidated analysis methods and reagents that do not reply the requirements of pharmacopoeias

2. Inconsistency in terms of sampling, analysis, fixing results

3. Incorrect concentration of the reagent on the surface of the test system.

1. Unsatisfactory quality of training

1. Violation of the expiration date

2. Violation of the storage conditions (temperature, lighting, packaging, air access)

1. Unsatisfactory quality of training

1. Error during sampling of substances

1. Invalid test result (false positive or false negative result)

2. Analysis without blank experiment

1. Failure to comply with the time period of observation

2. The absence of the blank experiment

3. Problems with fixing results (presence of impurities)

1. Unsatisfactory quality of training
Decrease of the quality of analysis. Invalid results of the analysis. Increasing expenses and time for production of test systems. Invalid results of the analysis.

The test system is unusable.

$$
\begin{array}{llll}
7 & 4 & 4 & 112
\end{array}
$$

$\begin{array}{llll}6 & 4 & 6 & 144\end{array}$

Invalid results of the analysis. The test system is unusable. Reduction of the test system 's expiration date. Decrease of the quality of analysis. Invalid results of the analysis. Increasing expenses and time for production of test-systems.

Invalid results of the analysis. The test system is unusable.

Reduction of the test system 's expiration date. Invalid results of the analysis. The test system is unusable.

Invalid results of the analysis.

Damage of a test-system. Leaking of substances being analyzed on skin, clothing, work surface.

$\begin{array}{llll}6 & 4 & 3 & 72\end{array}$

Invalid results of the analysis.

Invalid results of the analysis (high probability of false positive or false negative result).

$R P N($ ov. $)=3301$ 
Risk Assessment of Manufacturing and Usage of Test Systems for Quality Control of Compounded Preparations

Table 2. The priority number of risks at all the stages and phases of the lifecycle of test systems

\begin{tabular}{|c|c|c|c|c|c|}
\hline Stage (St.) & Phases (Ph.) & $\vec{z}_{\ddot{z}}^{\dot{m}}$ & 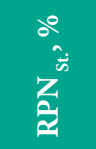 & 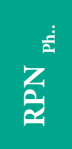 & 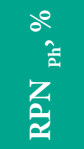 \\
\hline \multirow{8}{*}{$\begin{array}{l}\text { Manufacturing of the test } \\
\text { system }\end{array}$} & 1.1. Preparation of the workplace & \multirow{8}{*}{1814} & \multirow{8}{*}{54.95} & 135 & 4.09 \\
\hline & 1.2. Preparation of sheets of the filter paper & & & 262 & 7.94 \\
\hline & 1.3. Preparation of the reagent solution & & & 668 & 20.24 \\
\hline & 1.4. Physical immobilization & & & 282 & 8.54 \\
\hline & 1.5. Drying the test system & & & 138 & 4.18 \\
\hline & 1.6. Cutting of sheet into the test strips & & & 54 & 1.64 \\
\hline & 1.7. Packaging and labeling of the test systems & & & 191 & 5.79 \\
\hline & 1.8. Training of the personnel & & & 84 & 2.54 \\
\hline \multirow{3}{*}{$\begin{array}{l}\text { Quality control of the test } \\
\text { system }\end{array}$} & 2.1. Organoleptic analysis & \multirow{3}{*}{724} & \multirow{3}{*}{21.93} & 48 & 1.45 \\
\hline & 2.2. Chemical analysis & & & 592 & 17.93 \\
\hline & 2.3. Training of the personnel & & & 84 & 2.54 \\
\hline \multirow{2}{*}{ Storage } & 3.1. Control of the storage conditions & \multirow{2}{*}{147} & \multirow{2}{*}{4.45} & 105 & 3.18 \\
\hline & 3.2. Training of the personnel & & & 42 & 1.27 \\
\hline \multirow{4}{*}{ Using the test system } & 4.1. Sampling of substances & \multirow{4}{*}{616} & \multirow{4}{*}{18.66} & 72 & 2.18 \\
\hline & 4.2. Analysis & & & 156 & 4.73 \\
\hline & 4.3. Visual fixation of the results & & & 304 & 9.21 \\
\hline & 4.4. Training of the personnel & & & 84 & 2.54 \\
\hline
\end{tabular}

es can influence the expiration date of the test system;

$\diamond$ cutting of sheets into test strips (1.64\%) and organoleptic analysis (1.45\%) have low indexes RPN, due to the high probability of the detection of risks at this phase, and low impact of the above risks on quality control of compound preparations with the help of test system.

The Ways to Eliminate Possible Risks and Their Consequences

There are a number of different risks that affect the quality of the analysis using test systems. The following recommendations can be used to minimize these risks:

1. Development and validation of new methods of the quality control, using calibrated analytical equipment and reagents that comply with the requirements of the State Pharmacopoeia of Ukraine can minimize the risks at the phases of quality control of reagent solutions and test systems.
2. Introduction of the changes in the manufacturing process: simultaneous immobilization and drying of the series of test systems, can decrease risks associated with immobilization and drying time and also make it impossible to break and damage the sheets during their extraction from the reagent solution.

3. The proper control of packaging and storage conditions of test systems;

4. Improving the quality of training for work with test systems, development of standard operating procedures (SOPs) for work with these analytical tools.

\section{CONCLUSIONS}

Manufacturing, quality control, storage and use of test system based on filter paper impregnated with the salts of heavy metals are characterized by a large number of different risks that are able to have a negative impact on the results of chemical analysis of the compound preparations using test-systems. The data presented in the article allow systematizing and ap- 
Vadim Prokopets, Oleksandr Zdoryk, Viktoria Georgiyants

portioning risks and their consequences of the priority and developing recommendations to minimize the negative effects of these threats.

The quality control of the compounded preparations using test systems can significantly increase the following of the recommendations which are given in the article.

The list of recommendations is not final; the work on improving existing and developing new test systems is continued.

Improvement of the quality of these test kits allows incorporating these analytical tools into the list of the reagents (National part) that are recommended by the State Pharmacopoeia of Ukraine.

\section{REFERENCES}

1. Freedman ben-Horin S., Rousso T. Determination of Risk for Sterile Preparations. International journal of pharmaceutical compounding. 2014; 11, 1: 30-34.

2. Gudeman J., Jozwiakowski M., Chollet J., Randell M. Potential Risks of Pharmacy Compounding. Drugs in R\&D. 2013; 13, 1: 1-8.

3. ICH guideline $\mathrm{Q} 9$ on quality risk management EMA/INS/GMP/79766/2011. 2011: 20.

4. Prokopets V.V., Zdoryk O.A., Gura E.O. Dolibozhko N.V., Georgiyants V.A. The possibility for rapid analysis of water solutions of pyridoxine hydrochloride and sodium metamizol using test-kits with reagent iron (III). Ukrainian biopharmaceutical journal. 2014; 6, 35: 88-92.

5. Prokopets V.V., Zdoryk O.A., Georgiyants V.A. Development and application of test-kits for identification sulfanilamides in extemporal medicines. Collection of scientific works of staff members of NMAPE. 2015; 24, 5: 348-354.

6. Prokopets V.V., Zdoryk O.A., Georgiyants V.A. Application of test-kits with heavy metals salts for analysis of salts of benzoic and salicylic acids in extemporal medicines. Der Pharma Chemica. 2016: 8, 6: 122-128.

7. Hamid Mollah, Mike Long, Harold S. Baseman. Risk Management Applications in Pharmaceutical and Biopharmaceutical Manufacturing. Hoboken, New Jersey: John Wiley \& Sons, Inc.; 2013, 390.

8. Logunova L.N., Ustinova L.V. Evaluation of controllability and stability of business processes in a pharmaceutical organization. Far East Medical Journal. 2013; 4: 71-76.

9. Estorilio C., Posso R. The reduction of irregularities in the use of process FMEA. International Juornal of Quality \& Reliability Management. 2010; 6: 721-733.

10. Zdoryk O.A., Shtrimaitis O.V., Georgiyants V.A. Risk assessment of compounding and quality control of concentrated solutions in pharmacy conditions. News of Pharmacy. 2014; 1: 16-21. 